\title{
Distribution of Xanthomonas hortorum pv. carotae Populations in Naturally Infested Carrot Seed Lots
}

\author{
Jeness C. Scott and Jeremiah K. S. Dung ${ }^{\dagger}$ \\ Department of Botany and Plant Pathology, Central Oregon Agricultural Research and Extension Center, Oregon State \\ University, Madras, OR, U.S.A.
}

\begin{abstract}
Bacterial blight of carrot (Daucus carota subsp. sativus), caused by the plant-pathogenic bacterium Xanthomonas hortorum pv. carotae, is a common seedborne disease of carrot wherever the crop is grown. Carrot seed lots were evaluated to determine the variability and distribution of populations of $X$. hortorum pv. carotae among individual carrot seeds. Twentyfour carrot seed lots harvested between 2014 and 2016 were subjected to a bulk seed wash dilution-plate assay to obtain mean X. hortorum pv. carotae levels. Mean infestation levels resulting from the bulk seed wash assays among the 24 seed lots ranged from $1.2 \times 10^{7}$ and $9.6 \times 10^{8} \mathrm{CFU} / \mathrm{g}$ seed and averaged $3.6 \times 10^{8} \mathrm{CFU} / \mathrm{g}$ seed. Individual seeds from the same 24 lots were also tested with a scaled-down wash assay of individual seeds.

pv. carotae detection on individual seed in seed lots ranged from $0 \%$ (not detected) to $97.9 \%$, and the mean and median X. hortorum pv. carotae population on an individual seed was $8.3 \times 10^{4}$ and $6.3 \times 10^{1} \mathrm{CFU} / \mathrm{seed}$, respectively. Among individual seeds, X. hortorum pv. carotae populations ranged from 2 (the limit of detection of the assay) to $3.6 \times 10^{7}$ CFU/seed. CFU data for 23 of the 24 seed lots were nonnormal and the Log-Logistic (3P) distribution best described populations of X. hortorum pv. carotae recovered from individual carrot seeds. The influence and impact of nonnormal distributions of $X$. hortorum pv. carotae in commercial carrot seed lots on seed health tests, seedborne transmission, and bacterial blight epidemiology requires further study.
\end{abstract} Among the 1,380 seeds that were individually assayed, $475 \mathrm{X}$. hortorum pv. carotae-positive seeds were detected (34.4\%). Rates of X. hortorum
Keywords: pathogen detection, prokaryotes, seed crops
Bacterial blight of carrot (Daucus carota L. subsp. sativus Hoffm.), caused by the plant-pathogenic bacterium Xanthomonas hortorum pv. carotae Vauterin, Hoste, Kersters \& Swings 1995, was originally described as a disease in carrot seed production fields (Kendrick 1934) and is a common disease of carrot wherever the crop is grown (du Toit et al. 2014; Gugino et al. 2004; Myung et al. 2014; Nishiyama et al. 1979; Pruvost et al. 2010). Symptoms of bacterial blight include small, irregular, yellow lesions on foliage, stems, and petioles that can manifest into water-soaked, necrotic lesions and reduced root yields (Gilbertson 2002). During seed production, floral infections can result in blighted umbels, reduced seed yield, and reduced germination rates of harvested seed. The pathogen is also seedborne and seed transmitted (Umesh et al. 1998). Because $X$. hortorum pv. carotae is seedborne, it is a major concern not only for the hybrid carrot seed industry in the United States but also for regions that import carrot seed for fresh or processed carrot production.

Oregon is a major producer of hybrid carrot seed, which occurs on approximately 2,000 ha and exceeds $\$ 15$ million dollars in value (Butler and Simmons 2013). On average, the state produces 60\% of the seed used for carrot root crop production in the United States and up to $40 \%$ of the carrot seed supplied to the world. In the semiarid area of central Oregon, where carrot crops are grown for seed, bacterial blight symptoms can be inconspicuous but large epiphytic populations of $X$. hortorum pv. carotae can develop on otherwise healthy-appearing plants. At harvest, the seed from these plants can be infected or infested by $X$. hortorum pv. carotae, which can

${ }^{\dagger}$ Corresponding author: J. K. S. Dung; jeremiah.dung@oregonstate.edu

Funding: This project was funded by the Agricultural Research Foundation Competitive Grants Program and the Jefferson County Seed Growers Association.

The author(s) declare no conflict of interest.

Accepted for publication 24 February 2020.

C 2020 The American Phytopathological Society result in its transmission to new crops when the seed is planted (du Toit et al. 2005). Management of bacterial blight in seed production fields is expensive and often only partially effective at reducing pathogen levels in fields and seed lots.

Seed industry efforts to disinfest carrot seed lots of X. hortorum pv. carotae also are associated with high costs (Umesh et al. 1998). Hot water $\left(52^{\circ} \mathrm{C}\right.$ for $\left.25 \mathrm{~min}\right)$ is a common treatment, which can be effective but does not always entirely eradicate the pathogen. Hot water treatments can also reduce germination or the shelf life of seed lots if performed improperly (Miller and Lewis Ivey, 2006). Germination can be reduced further if multiple treatments are needed to reduce bacterial levels below the $1 \times 10^{5}$ bacteria/g of seed threshold that was established for carrot seed planted in the Central Valley of California (Umesh et al. 1998).

Considerations when developing a seed health assay can include the desired tolerance standard (maximum percentage of infested seed that is acceptable) and the detection limit of the assay (minimum number of pathogens per seed that can be detected). These parameters would inform the determination of both sample (total number of seeds) and subsample size to be tested in each assay and to help determine seedborne disease transmission risk. Bulk samples of carrot seed are tested for $X$. hortorum pv. carotae using a dilutionplating protocol (Asma 2005) that involves soaking three 10-g samples of a seed lot (each approximately 10,000 seeds) in a buffer, followed by serial dilution plating the wash onto a semiselective culture medium (Asma 2005). The assay is designed to reliably measure a mean level of infestation with sufficient precision near the accepted risk threshold of $1 \times 10^{5} \mathrm{bacteria} / \mathrm{g}$ of seed.

Many testing protocols assume that infestation levels are relatively uniform among individual seeds (Russell 1988). However, some studies contradict this assumption. For example, Dutta et al. (2013) observed that the number of $X$. axonopodis pv. vignicola, Clavibacter michiganensis subsp. michiganensis, X. euvesicatoria, Acidivorax citrulli, and Pantoea stewartii subsp. stewartii recovered from individual cowpea, tomato, pepper, watermelon, and corn seed, respectively, varied over several orders of magnitude and that these per-seed estimates of infestation size followed a nonnormal distribution. If the distribution of $X$. hortorum pv. carotae among infested carrot seed in a seed lot is nonnormal, assay results from bulk samples could result in an inaccurate estimate of transmission risk when 
seedborne $X$. hortorum pv. carotae serves as primary inoculum. For example, a relatively few but highly infested seeds in a seed lot could initiate a bacterial blight epidemic but perhaps viable seedlings fail to emerge from these seed. Likewise, the spatial and temporal dynamics of bacterial blight incidence may differ in fields planted to seed lots with a nonnormal distribution of the pathogen compared with fields planted with a uniformly infested seed lot. Considerations for seed production include the sample size required for seed health tests and the effectiveness of seed treatments to reduce large populations on a small number of seeds in a seed lot. The objective of this study was to determine the variability and distribution of $X$. hortorum pv. carotae populations among individual seeds in naturally infested carrot seed lots produced in different years.

\section{Materials and Methods}

Bulk seed lot assays. In total, 24 seed lots were obtained from commercial carrot seed producers in Madras, OR in $2014(n=10)$, $2015(n=7)$, and $2016(n=7)$. Seed lots were cleaned and conditioned but not hot water treated prior to sampling. Seed lots were subjected to a seed wash dilution-plate assay to determine the mean $X$. hortorum pv. carotae infestation (CFU per gram). The seed wash dilution-plate assay was based on the most recent International Seed Testing Association-validated protocol (Asma 2005). Briefly, three 10 -g subsamples from each seed lot were soaked for $2 \mathrm{~h}$ at room temperature $\left(23^{\circ} \mathrm{C}\right)$ in a $250-\mathrm{ml}$ flask containing $100 \mathrm{ml}$ of sterile $0.0125 \mathrm{M} \mathrm{PO}_{4}$ buffer $\left(16.1 \mathrm{ml}\right.$ of $0.5 \mathrm{M} \mathrm{KH}_{2} \mathrm{PO}_{4}$ and $8.9 \mathrm{ml}$ of $0.5 \mathrm{M} \mathrm{K}_{2} \mathrm{HPO}_{4}$ in $975 \mathrm{ml}$ of water) and $35 \mu \mathrm{l}$ of Tween 20 (PBT). After the soak, the flasks were placed on a horizontal orbital shaker set at $250 \mathrm{rpm}$ for $5 \mathrm{~min}$. A 10 -fold dilution series ranging from $10^{\circ}$ to $10^{-5}$ concentration was prepared for each seed wash by mixing with sterile PBT. A 0.1-ml aliquot of each dilution was spread plated onto two replicate $9-\mathrm{cm}$ Petri plates that contained semi-selective XCS agar medium (Williford and Schaad 1984). Plates were incubated at $28^{\circ} \mathrm{C}$ in the dark and monitored for the development of colonies with a morphological appearance typical of $X$. hortorum pv. carotae. The number of $X$. hortorum pv. carotae colonies was counted after 5 to 7 days of incubation. Suspect colonies were subcultured onto yeast extract-dextrose-calcium carbonate (YDC) agar medium (Starr and Stephens 1964) and subjected to a species-specific PCR assay (Meng et al. 2004) to confirm their identities.

Assays of individual seeds. Individual seeds were assayed with a modified dilution plating assay. Single seeds were placed individually into sterile 8-strip, $0.3-\mathrm{ml}$ PCR tubes that contained $0.2 \mathrm{ml}$ of PBT. Seed were soaked for $2 \mathrm{~h}$ at room temperature. The seedcontaining tubes were attached horizontally to a platform vortex and agitated for $5 \mathrm{~min}$. The wash from each tube was serially diluted 10 -fold to $10^{-7}$ in sterile PBT. Aliquots $(0.1 \mathrm{ml})$ of each dilution were spread on a single plate of XCS and incubated at $28^{\circ} \mathrm{C}$ as described above. Individual seeds were processed until a minimum of 25 positive seeds were identified from each of at least 10 seed lots. The infested seed were treated as a subset of positive seed from the parent seed lot and the bacterial populations recovered were used for analysis.

Data analyses. The Shapiro-Wilk test (Shapiro and Wilk 1965) was used to test for normality of nontransformed and $\log _{10^{-}}$ tranformed estimates of $X$. hortorum pv. carotae CFU per seed. If conditions for normality were not met, then the data were examined with a probability distribution estimation program (EasyFit ver.5.6; Mathwave Technologies, Dnepropetrovsk, Ukraine). The best fit of the data to various probability distributions was determined by comparing Anderson-Darling and Kolmogorov-Smirnov goodness-of-fit test statistics and visual examination of histograms and cumulative probability plots.

\section{Results}

Using the 10-g bulk seed wash assay, 24 commercial carrot seed lots were tested and all were shown to be at or above $1 \times 10^{4}$ $\mathrm{CFU} / \mathrm{g}$ seed with the bulk $(10-\mathrm{g})$ seed wash assay. The average infestation level among seed lots was $3.6 \times 10^{8} \mathrm{CFU} / \mathrm{g}$ seed, with mean infestation levels of $1.2 \times 10^{7}, 9.6 \times 10^{8}$, and $2.5 \times 10^{8} \mathrm{CFU} / \mathrm{g}$ seed in 2014, 2015, and 2016, respectively (Table 1).
In total, 1,380 seeds were assayed individually with $X$. hortorum pv. carotae detected on 475 seeds (34.4\%). With this assay method, individual seed infestation rates ranged from $0 \%$ (no infested seed detected) to $97.9 \%$ infested seed per seed lot (Table 2). The mean infestation of individual seeds was $8.3 \times 10^{4} \mathrm{CFU}$, which was 1,300 times greater than the median infestation of $6.3 \times 10^{1} \mathrm{CFU} / \mathrm{seed}$. Among individual seeds on which $X$. hortorum pv. carotae was detected, the infestation size ranged from $2 \mathrm{CFU} /$ seed (assay detection limit) to $3.6 \times 10^{7} \mathrm{CFU} / \mathrm{seed}$. Of the 24 seed lots, 8 contained individual seeds with $>10^{5} \mathrm{CFU}$ (Table 3 ).

Data for all seed lots, with the exception of one (sample 10), were nonnormal, and most exhibited large skewness and kurtosity values (Table 4). Skewness values ranged from 0.90 to 6.49 for nontransformed data, with a mean value of 3.65. Kurtosis values for nontransformed data ranged from -0.91 to 42.34 , with a mean kurtosis value of 16.08 among the 24 seed lots. $\log _{10}$-transformation of the data resulted in normal distributions $(P>0.05)$ for two samples (samples 6 and 10) and reduced skewness and kurtosis values for all samples; however, $\log _{10}$-transformation resulted in negative kurtosis values for six seed lots, indicating smaller tails than expected in a normal distribution.

Fifty continuous and discrete probability distributions were examined for the 475 infested seeds collected from 24 seed lots. The distribution that provided the best fit for the entire dataset was the Log-Logistic (3P) model. The distributions that provided the best fits for individual seed lots included the Burr, Weibull, and Log-Pearson 3 distributions (Table 5), all of which are highly skewed.

\section{Discussion}

Carrot seed lots are routinely screened for X. hortorum pv. carotae after harvest and subsequent conditioning operations to determine whether hot water or chemical treatment is necessary. In most cases, seed lots with no detectable $X$. hortorum pv. carotae are desired and seed lots require some form of postharvest treatment, which is costly and can have negative effects on seed quality. A better understanding of seed lot infestation and, in particular, the incidence and level of $X$. hortorum pv. carotae on individual seeds is important considering the cost associated with screening and treating large volumes of seed. The results from this study indicate that $X$. hortorum pv. carotae populations on individual carrot seeds do not follow normal distributions. The population distributions observed in this study among seed lots often exhibited vastly different mean and median values, with inflated mean values due to the influence of a small proportion of highly infested seed. The largest $X$. hortorum pv. carotae population observed on an individual seed in this study was $3.6 \times 10^{7} \mathrm{CFU}$;

Table 1. Nontransformed and $\log _{10}$-transformed Xanthomonas hortorum pv. carotae populations recovered from commercial carrot seed lots subjected to seed wash assays of bulk (10-g) samples and from assays of individual seeds

\begin{tabular}{|c|c|c|c|c|c|c|}
\hline \multirow[b]{3}{*}{ Year } & \multirow[b]{3}{*}{$N^{\mathbf{a}}$} & \multirow{2}{*}{\multicolumn{2}{|c|}{$\begin{array}{c}\text { Bulk (10-g) seed } \\
\text { assay }^{b}\end{array}$}} & \multicolumn{3}{|c|}{ Individual seed assay } \\
\hline & & & & \multirow{2}{*}{$\begin{array}{l}\text { Pos } \\
(\%)\end{array}$} & \multirow[b]{2}{*}{ Mean } & \multirow[b]{2}{*}{$\operatorname{Max}$} \\
\hline & & Mean & SD & & & \\
\hline \multicolumn{7}{|c|}{ Nontransformed } \\
\hline 2014 & 10 & $1.17 \mathrm{E}+07$ & $4.02 \mathrm{E}+06$ & $34.0 \%$ & $4.70 \mathrm{E}+03$ & $1.92 \mathrm{E}+05$ \\
\hline 2015 & 7 & $9.56 \mathrm{E}+08$ & $1.56 \mathrm{E}+09$ & $26.4 \%$ & $3.79 \mathrm{E}+04$ & $9.22 \mathrm{E}+05$ \\
\hline 2016 & 7 & $2.47 \mathrm{E}+08$ & $1.19 \mathrm{E}+06$ & $60.2 \%$ & $1.92 \mathrm{E}+05$ & $7.33 \mathrm{E}+06$ \\
\hline Total & 24 & $3.56 \mathrm{E}+08$ & $4.57 \mathrm{E}+08$ & $39.4 \%$ & $6.89 \mathrm{E}+04$ & $2.49 \mathrm{E}+06$ \\
\hline \multicolumn{7}{|c|}{$\begin{array}{l}\log _{10^{-}} \\
\text {transformed }\end{array}$} \\
\hline 2014 & 10 & 6.64 & 6.29 & $34.0 \%$ & 2.05 & 3.50 \\
\hline 2015 & 7 & 6.71 & 6.80 & $26.4 \%$ & 2.13 & 3.67 \\
\hline 2016 & 7 & 8.03 & 5.20 & $60.2 \%$ & 3.82 & 5.32 \\
\hline Total & 24 & 7.07 & 6.12 & $39.4 \%$ & 2.63 & 4.12 \\
\hline
\end{tabular}

a Number of seed lots.

b Mean $=$ mean CFU per gram of seed and SD $=$ standard deviation.

c In total, 461, 485, and 434 seeds were tested in 2014, 2015, and 2016 , respectively. Pos $=$ percentage of positive seeds, Mean $=$ mean CFU per individual seed, and Max = maximum CFU per individual seed 
at this infestation level, it would only take 10 infested seeds per gram of seed to explain the infestation level observed within this seed lot $\left(4.6 \times 10^{8} \mathrm{CFU} / \mathrm{g}\right)$. For seed lots with highly infested seed present,

Table 2. Nontransformed and $\log _{10}$-transformed Xanthomonas hortorum $\mathrm{pv}$. carotae populations recovered from individual commercial carrot seed lots subjected to seed wash assays of bulk (10-g) samples and the number of infested seeds as determined in assays of individual seeds

\begin{tabular}{|c|c|c|c|c|}
\hline \multirow[b]{2}{*}{ Year, seed lot } & \multicolumn{2}{|c|}{ Bulk (10-g) seed assay ${ }^{a}$} & \multicolumn{2}{|c|}{$\begin{array}{c}\text { Individual } \\
\text { seed assayb }\end{array}$} \\
\hline & Mean & SD & $N$ & Pos $(\%$ \\
\hline \multirow{2}{*}{\multicolumn{5}{|c|}{$\begin{array}{l}\text { Nontransformed } \\
2014\end{array}$}} \\
\hline & & & & \\
\hline 1 & $1.6 \mathrm{E}+07$ & $6.6 \mathrm{E}+06$ & 56 & $30(54)$ \\
\hline 2 & $1.1 \mathrm{E}+05$ & $9.3 \mathrm{E}+04$ & 51 & $4(8)$ \\
\hline 3 & $1.4 \mathrm{E}+07$ & $3.4 \mathrm{E}+06$ & 35 & $33(94)$ \\
\hline 4 & $3.5 \mathrm{E}+07$ & $2.8 \mathrm{E}+06$ & 50 & $28(56)$ \\
\hline 5 & $1.3 \mathrm{E}+07$ & $1.1 \mathrm{E}+07$ & 46 & $12(26)$ \\
\hline 6 & $1.2 \mathrm{E}+06$ & $8.6 \mathrm{E}+05$ & 30 & $4(13)$ \\
\hline 7 & $1.4 \mathrm{E}+07$ & $6.1 \mathrm{E}+06$ & 91 & $7(8)$ \\
\hline 8 & $1.2 \mathrm{E}+07$ & $4.3 \mathrm{E}+06$ & 30 & $7(23)$ \\
\hline 9 & $1.0 \mathrm{E}+07$ & $4.8 \mathrm{E}+06$ & 42 & $16(38)$ \\
\hline 10 & $1.2 \mathrm{E}+05$ & $1.1 \mathrm{E}+05$ & 30 & $6(20)$ \\
\hline \multicolumn{5}{|l|}{2015} \\
\hline 11 & $6.7 \mathrm{E}+09$ & $1.1 \mathrm{E}+10$ & 63 & $50(79)$ \\
\hline 12 & $7.6 \mathrm{E}+06$ & $2.4 \mathrm{E}+06$ & 97 & $26(27)$ \\
\hline 13 & $7.6 \mathrm{E}+05$ & $5.5 \mathrm{E}+05$ & 87 & $8(9)$ \\
\hline 14 & $1.7 \mathrm{E}+07$ & $1.6 \mathrm{E}+07$ & 30 & $18(60)$ \\
\hline 15 & $1.7 \mathrm{E}+06$ & $1.1 \mathrm{E}+06$ & 64 & $6(9)$ \\
\hline 16 & $2.4 \mathrm{E}+04$ & $2.4 \mathrm{E}+06$ & 48 & ND \\
\hline 17 & $3.7 \mathrm{E}+06$ & $6.5 \mathrm{E}+05$ & 96 & ND \\
\hline \multicolumn{5}{|l|}{2016} \\
\hline 18 & $5.5 \mathrm{E}+05$ & $8.2 \mathrm{E}+05$ & 88 & $15(17)$ \\
\hline 19 & $2.1 \mathrm{E}+08$ & $4.2 \mathrm{E}+05$ & 72 & $31(43)$ \\
\hline 20 & $3.2 \mathrm{E}+08$ & $3.1 \mathrm{E}+01$ & 18 & $10(56)$ \\
\hline 21 & $4.6 \mathrm{E}+08$ & $5.2 \mathrm{E}+06$ & 48 & $43(90)$ \\
\hline 22 & $1.4 \mathrm{E}+08$ & $3.5 \mathrm{E}+05$ & 48 & 47 (98) \\
\hline 23 & $4.6 \mathrm{E}+08$ & $1.4 \mathrm{E}+06$ & 48 & $44(92)$ \\
\hline 24 & $1.3 \mathrm{E}+08$ & $9.1 \mathrm{E}+04$ & 112 & $30(27)$ \\
\hline \multicolumn{5}{|c|}{$\begin{array}{l}\log _{10} \text {-transformed } \\
\quad 2014\end{array}$} \\
\hline 1 & 7.21 & 6.82 & 56 & $30(54)$ \\
\hline 2 & 5.02 & 4.97 & 51 & $4(8)$ \\
\hline 3 & 7.16 & 6.53 & 35 & $33(94)$ \\
\hline 4 & 7.55 & 6.44 & 50 & $28(56)$ \\
\hline 5 & 7.11 & 7.05 & 46 & $12(26)$ \\
\hline 6 & 6.06 & 5.93 & 30 & $4(13)$ \\
\hline 7 & 7.14 & 6.78 & 91 & $7(8)$ \\
\hline 8 & 7.08 & 6.63 & 30 & $7(23)$ \\
\hline 9 & 7.02 & 6.68 & 42 & $16(38)$ \\
\hline 10 & 5.06 & 5.05 & 30 & $6(20)$ \\
\hline \multicolumn{5}{|l|}{2015} \\
\hline 11 & 9.82 & 10.04 & 63 & $50(79)$ \\
\hline 12 & 6.88 & 6.38 & 97 & $26(27)$ \\
\hline 13 & 5.88 & 5.74 & 87 & $8(9)$ \\
\hline 14 & 7.23 & 7.20 & 30 & $18(60)$ \\
\hline 15 & 6.24 & 6.05 & 64 & $6(9)$ \\
\hline 16 & 4.39 & 6.38 & 48 & ND \\
\hline 17 & 6.56 & 5.81 & 96 & ND \\
\hline \multicolumn{5}{|l|}{2016} \\
\hline 18 & 5.74 & 5.91 & 88 & 15 (17) \\
\hline 19 & 8.31 & 5.63 & 72 & $31(43)$ \\
\hline 20 & 8.51 & 1.49 & 18 & $10(56)$ \\
\hline 21 & 8.67 & 6.72 & 48 & $43(90)$ \\
\hline 22 & 8.16 & 5.55 & 48 & 47 (98) \\
\hline 23 & 8.66 & 6.15 & 48 & $44(92)$ \\
\hline 24 & 8.13 & 4.96 & 112 & $30(27)$ \\
\hline
\end{tabular}

a Mean = mean CFU per gram of seed and SD = standard deviation

${ }^{\mathrm{b}} N=$ number of seed tested, Pos = number and percentage of positive seeds, and ND $=$ not detected. the bulk seed wash assay only demonstrates the detection of these highly infested seeds, because seeds that are infested at lower levels do not contribute enough bacteria to affect the mean CFU per gram. Regardless, the presence of a few, highly infested seeds in a seed lot may result in an unacceptable level of $X$. hortorum pv. carotae in a bulk seed wash test and can negatively impact seed producers and seed lot marketability.

The bulk seed wash assay measures the mean infestation level but does not differentiate between a high incidence of seed infested at a low- to moderate level or a low frequency of seed that are highly infested. This lack of differentiation contributes to the discrepancy

Table 3. Summary statistics and variation for nontransformed and $\log _{10^{-}}$ transformed populations of Xanthomonas hortorum pv. carotae recovered from individual carrot seeds ${ }^{\mathrm{a}}$

\begin{tabular}{|c|c|c|c|c|c|c|}
\hline Year, seed lot & $N^{\mathbf{b}}$ & Minimum & Maximum & Mean & Median & $\mathbf{S D}^{\mathbf{c}}$ \\
\hline \multicolumn{7}{|l|}{$\begin{array}{l}\text { Nontransformed } \\
2014\end{array}$} \\
\hline 1 & 30 & $2.0 \mathrm{E}+00$ & $1.5 \mathrm{E}+04$ & $6.0 \mathrm{E}+02$ & $6.0 \mathrm{E}+00$ & $2.7 \mathrm{E}+03$ \\
\hline 2 & 4 & $2.0 \mathrm{E}+00$ & $2.0 \mathrm{E}+00$ & $2.0 \mathrm{E}+00$ & $2.0 \mathrm{E}+00$ & $0.0 \mathrm{E}+00$ \\
\hline 3 & 33 & $2.0 \mathrm{E}+00$ & $5.4 \mathrm{E}+05$ & $1.6 \mathrm{E}+04$ & $7.0 \mathrm{E}+01$ & $9.4 \mathrm{E}+04$ \\
\hline 4 & 28 & $2.0 \mathrm{E}+00$ & $1.2 \mathrm{E}+06$ & $4.6 \mathrm{E}+04$ & $6.0 \mathrm{E}+00$ & $2.3 \mathrm{E}+05$ \\
\hline 5 & 12 & $2.0 \mathrm{E}+00$ & $1.1 \mathrm{E}+03$ & $1.6 \mathrm{E}+02$ & $4.0 \mathrm{E}+00$ & $3.4 \mathrm{E}+02$ \\
\hline 6 & 4 & $2.0 \mathrm{E}+00$ & $3.0 \mathrm{E}+01$ & $1.0 \mathrm{E}+01$ & $4.0 \mathrm{E}+00$ & $1.3 \mathrm{E}+01$ \\
\hline 7 & 7 & $2.0 \mathrm{E}+00$ & $4.2 \mathrm{E}+04$ & $6.1 \mathrm{E}+03$ & $4.0 \mathrm{E}+00$ & $1.6 \mathrm{E}+04$ \\
\hline 8 & 7 & $2.0 \mathrm{E}+00$ & $9.6 \mathrm{E}+04$ & $2.2 \mathrm{E}+04$ & $2.0 \mathrm{E}+00$ & $3.9 \mathrm{E}+04$ \\
\hline 9 & 16 & $2.0 \mathrm{E}+00$ & $1.3 \mathrm{E}+03$ & $1.3 \mathrm{E}+02$ & $1.1 \mathrm{E}+01$ & $3.4 \mathrm{E}+02$ \\
\hline 10 & 6 & $2.0 \mathrm{E}+00$ & $2.8 \mathrm{E}+01$ & $1.1 \mathrm{E}+01$ & $6.0 \mathrm{E}+00$ & $1.1 \mathrm{E}+01$ \\
\hline \multicolumn{7}{|l|}{2015} \\
\hline 11 & 50 & $2.0 \mathrm{E}+00$ & $6.4 \mathrm{E}+06$ & $3.2 \mathrm{E}+05$ & $1.2 \mathrm{E}+03$ & $1.3 \mathrm{E}+06$ \\
\hline 12 & 26 & $2.0 \mathrm{E}+00$ & $5.2 \mathrm{E}+04$ & $4.2 \mathrm{E}+03$ & $2.2 \mathrm{E}+01$ & $1.2 \mathrm{E}+04$ \\
\hline 13 & 8 & $2.0 \mathrm{E}+00$ & $1.1 \mathrm{E}+02$ & $3.3 \mathrm{E}+01$ & $1.2 \mathrm{E}+01$ & $4.0 \mathrm{E}+01$ \\
\hline 14 & 18 & $2.0 \mathrm{E}+00$ & $1.6 \mathrm{E}+03$ & $1.7 \mathrm{E}+02$ & $9.0 \mathrm{E}+00$ & $4.3 \mathrm{E}+02$ \\
\hline 15 & 6 & $2.0 \mathrm{E}+00$ & $3.6 \mathrm{E}+01$ & $9.0 \mathrm{E}+00$ & $4.0 \mathrm{E}+00$ & $1.3 \mathrm{E}+01$ \\
\hline 16 & 0 & $0.0 \mathrm{E}+00$ & $0.0 \mathrm{E}+00$ & $0.0 \mathrm{E}+00$ & $0.0 \mathrm{E}+00$ & $0.0 \mathrm{E}+00$ \\
\hline 17 & 0 & $0.0 \mathrm{E}+00$ & $0.0 \mathrm{E}+00$ & $0.0 \mathrm{E}+00$ & $0.0 \mathrm{E}+00$ & $0.0 \mathrm{E}+00$ \\
\hline \multicolumn{7}{|c|}{ (1) } \\
\hline 18 & 15 & $2.0 \mathrm{E}+00$ & $7.2 \mathrm{E}+01$ & $9.5 \mathrm{E}+00$ & $2.0 \mathrm{E}+00$ & $1.8 \mathrm{E}+01$ \\
\hline 19 & 31 & $2.0 \mathrm{E}+00$ & $3.6 \mathrm{E}+06$ & $1.2 \mathrm{E}+05$ & $8.0 \mathrm{E}+00$ & $6.5 \mathrm{E}+05$ \\
\hline 20 & 10 & $2.0 \mathrm{E}+00$ & $1.3 \mathrm{E}+02$ & $2.1 \mathrm{E}+01$ & $7.0 \mathrm{E}+00$ & $4.0 \mathrm{E}+01$ \\
\hline 21 & 43 & $2.0 \mathrm{E}+00$ & $3.6 \mathrm{E}+07$ & $9.4 \mathrm{E}+05$ & $4.6 \mathrm{E}+01$ & $5.5 \mathrm{E}+06$ \\
\hline 22 & 47 & $4.0 \mathrm{E}+00$ & $2.4 \mathrm{E}+06$ & $6.9 \mathrm{E}+04$ & $5.0 \mathrm{E}+01$ & $3.6 \mathrm{E}+05$ \\
\hline 23 & 44 & $2.0 \mathrm{E}+00$ & $8.4 \mathrm{E}+06$ & $3.9 \mathrm{E}+05$ & $2.3 \mathrm{E}+01$ & $1.5 \mathrm{E}+06$ \\
\hline 24 & 30 & $2.0 \mathrm{E}+00$ & $7.4 \mathrm{E}+05$ & $5.9 \mathrm{E}+04$ & $3.4 \mathrm{E}+01$ & $1.7 \mathrm{E}+05$ \\
\hline \multicolumn{7}{|l|}{$\begin{array}{l}\log _{10^{-}} \\
\text {transformed } \\
2014\end{array}$} \\
\hline 1 & 30 & 0.48 & 4.18 & 1.17 & 0.85 & 0.89 \\
\hline 2 & 4 & 0.48 & 0.48 & 0.48 & 0.48 & 0.00 \\
\hline 3 & 33 & 0.48 & 5.73 & 1.75 & 1.85 & 0.97 \\
\hline 4 & 28 & 0.48 & 6.09 & 1.26 & 0.85 & 1.30 \\
\hline 5 & 12 & 0.48 & 3.05 & 1.13 & 0.70 & 0.99 \\
\hline 6 & 4 & 0.48 & 1.49 & 0.82 & 0.66 & 0.48 \\
\hline 7 & 7 & 0.48 & 4.62 & 1.52 & 0.70 & 1.61 \\
\hline 8 & 7 & 0.48 & 4.98 & 1.76 & 0.48 & 2.13 \\
\hline 9 & 16 & 0.48 & 3.13 & 1.24 & 1.08 & 0.80 \\
\hline 10 & 6 & 0.48 & 1.46 & 0.88 & 0.76 & 0.46 \\
\hline \multicolumn{7}{|l|}{2015} \\
\hline 11 & 50 & 0.48 & 6.81 & 2.90 & 3.06 & 1.90 \\
\hline 12 & 26 & 0.48 & 4.72 & 1.75 & 1.36 & 1.28 \\
\hline 13 & 8 & 0.48 & 2.05 & 1.17 & 1.06 & 0.63 \\
\hline 14 & 18 & 0.48 & 3.20 & 1.27 & 1.00 & 0.87 \\
\hline 15 & 6 & 0.48 & 1.57 & 0.79 & 0.70 & 0.41 \\
\hline 16 & ND & ND & ND & ND & ND & ND \\
\hline 17 & ND & ND & ND & ND & ND & ND \\
\hline \multicolumn{7}{|l|}{2016} \\
\hline 18 & 15 & 0.48 & 1.86 & 0.76 & 0.48 & 0.41 \\
\hline 19 & 31 & 0.48 & 6.56 & 1.31 & 0.95 & 1.32 \\
\hline 20 & 10 & 0.48 & 2.13 & 0.95 & 0.87 & 0.54 \\
\hline 21 & 43 & 0.48 & 7.56 & 2.17 & 1.67 & 1.65 \\
\hline 22 & 47 & 0.70 & 6.38 & 2.16 & 1.71 & 1.34 \\
\hline 23 & 44 & 0.48 & 6.92 & 2.09 & 1.38 & 1.83 \\
\hline 24 & 30 & 0.48 & 5.87 & 2.17 & 1.46 & 1.83 \\
\hline
\end{tabular}

a $\mathrm{ND}=$ not determined.

b Number of Xanthomonas hortorum pv. carotae-positive seeds detected in the seed lot.

c Standard deviation. 
observed in mean infestation levels between the bulk wash and individual seed wash assays. Assuming 1,000 seeds/g, the mean infestation of the seed lots based on the bulk (10-g) assay would be expected to be 1,000 times greater than the mean infestation level observed for individual seeds. Contributing to this discrepancy between the bulk and individual infestation levels is likely the lot-to-lot variation in

Table 4. Shapiro-Wilk statistic $(W)$, skewness, and kurtosis values of nontransformed and $\log _{10}$-transformed populations of Xanthomonas hortorum pv. carotae recovered from individual carrot seeds ${ }^{\mathrm{a}}$

\begin{tabular}{|c|c|c|c|c|c|}
\hline Year, seed lot & $N^{\mathbf{b}}$ & $W$ & $P$ value & Skewness & Kurtosis \\
\hline \multicolumn{6}{|l|}{ Nontransformed } \\
\hline \multicolumn{6}{|l|}{2014} \\
\hline 1 & 30 & 0.22 & $<0.0001$ & 5.37 & 29.17 \\
\hline 2 & 4 & ND & ND & ND & ND \\
\hline 3 & 33 & 0.17 & $<0.0001$ & 5.74 & 33.00 \\
\hline 4 & 28 & 0.21 & $<0.0001$ & 5.26 & 27.77 \\
\hline 5 & 12 & 0.56 & $<0.0001$ & 2.47 & 6.25 \\
\hline 6 & 4 & 0.73 & 0.0238 & 1.89 & 3.58 \\
\hline 7 & 7 & 0.46 & $<0.0001$ & 2.64 & 6.99 \\
\hline 8 & 7 & 0.65 & 0.0011 & 1.55 & 1.13 \\
\hline 9 & 16 & 0.43 & $<0.0001$ & 3.50 & 12.75 \\
\hline 10 & 6 & 0.82 & 0.0949 & 0.90 & -0.91 \\
\hline \multicolumn{6}{|l|}{2015} \\
\hline 11 & 50 & 0.27 & $<0.0001$ & 4.64 & 20.93 \\
\hline 12 & 26 & 0.43 & $<0.0001$ & 3.31 & 11.90 \\
\hline 13 & 8 & 0.81 & 0.0355 & 1.21 & 0.47 \\
\hline 14 & 18 & 0.45 & $<0.0001$ & 2.88 & 7.73 \\
\hline 15 & 6 & 0.60 & 0.0004 & 2.38 & 5.73 \\
\hline 16 & 0 & ND & ND & ND & ND \\
\hline 17 & 0 & ND & ND & ND & ND \\
\hline \multicolumn{6}{|l|}{2016} \\
\hline 18 & 15 & 0.47 & $<0.0001$ & 3.46 & 12.59 \\
\hline 19 & 31 & 0.18 & $<0.0001$ & 5.56 & 30.96 \\
\hline 20 & 10 & 0.50 & $<0.0001$ & 3.01 & 9.27 \\
\hline 21 & 43 & 0.16 & $<0.0001$ & 6.49 & 42.34 \\
\hline 22 & 47 & 0.20 & $<0.0001$ & 6.40 & 42.33 \\
\hline 23 & 44 & 0.30 & $<0.0001$ & 4.72 & 23.40 \\
\hline 24 & 30 & 0.41 & $<0.0001$ & 3.23 & 10.23 \\
\hline \multicolumn{6}{|c|}{$\begin{array}{l}\log _{10} \text {-transformed } \\
2014\end{array}$} \\
\hline 1 & 30 & 0.74 & $<0.0001$ & 2.06 & 4.17 \\
\hline 2 & 4 & ND & ND & ND & ND \\
\hline 3 & 33 & 0.82 & $<0.0001$ & 2.07 & 8.16 \\
\hline 4 & 28 & 0.60 & $<0.0001$ & 2.85 & 8.31 \\
\hline 5 & 12 & 0.68 & 0.0005 & 1.33 & -0.06 \\
\hline 6 & 4 & 0.84 & 0.1884 & 1.32 & 1.04 \\
\hline 7 & 7 & 0.74 & 0.0088 & 1.56 & 1.47 \\
\hline 8 & 7 & 0.63 & 0.0006 & 1.23 & -0.82 \\
\hline 9 & 16 & 0.86 & 0.0188 & 1.15 & 0.73 \\
\hline 10 & 6 & 0.81 & 0.0705 & 0.33 & -2.51 \\
\hline \multicolumn{6}{|l|}{2015} \\
\hline 11 & 50 & 0.91 & 0.0012 & 0.33 & -1.10 \\
\hline 12 & 26 & 0.81 & 0.0002 & 1.33 & 0.66 \\
\hline 13 & 8 & 0.89 & 0.2304 & 0.23 & -1.94 \\
\hline 14 & 18 & 0.84 & 0.0064 & 1.16 & 0.43 \\
\hline 15 & 6 & 0.79 & 0.0468 & 1.80 & 3.60 \\
\hline 16 & 0 & ND & ND & ND & ND \\
\hline 17 & 0 & ND & ND & ND & ND \\
\hline \multicolumn{6}{|l|}{2016} \\
\hline 18 & 15 & 0.75 & 0.0008 & 1.65 & 2.61 \\
\hline 19 & 31 & 0.62 & $<0.0001$ & 2.91 & 9.25 \\
\hline 20 & 10 & 0.83 & 0.0379 & 1.12 & 1.20 \\
\hline 21 & 43 & 0.82 & $<0.0001$ & 1.69 & 2.68 \\
\hline 22 & 47 & 0.81 & $<0.0001$ & 1.64 & 2.17 \\
\hline 23 & 44 & 0.70 & $<0.0001$ & 1.69 & 1.48 \\
\hline 24 & 30 & 0.84 & 0.0003 & 0.82 & -0.75 \\
\hline
\end{tabular}

a $\mathrm{ND}=$ not determined

b Number of Xanthomonas hortorum pv. carotae-positive seeds detected in the seed lot. seed weight. For the lots that we assayed, 100 seed weights were between 0.125 and $0.290 \mathrm{~g}$, resulting in 800 and $345 \mathrm{seeds} / \mathrm{g}$, respectively (data not shown). Additionally, our assay was designed to examine the distribution of the pathogen on individual seeds, not to test enough individual seeds to accurately represent the mean infestation levels of the entire seed lot. For some lots, depending on the variation of seed-to-seed infestation rates and incidence of infested seed, the number of individual seeds that would have to be assayed would likely be near 10,000 seeds, or the number used in the bulk seed assay protocol.

The distributions observed in this study, including the log-logistic (three-parameter) distribution that best described the entire data set, are asymmetric and positively skewed. Dutta et al. (2013) assayed naturally infested seed and found that populations of several different phytopathogenic bacteria, including $X$. axonopodis pv. vignicola and $X$. euvesicatoria, were highly skewed and fit various nonnormal distributions. Populations of bacteria on leaves and in the rhizome have also been described as fitting log-normal or nonnormal distributions (Hirano et al. 1982; Ishimaru et al. 1991; Loper et al. 1984). The variability of phytopathogenic bacterial populations among individual seeds, leaves, or rhizospheres is likely influenced by plant species and cultivar, plant phenology, weather and microclimates, and the microbiome at small spatial scales.

The highly skewed distributions of $X$. hortorum pv. carotae in commercial carrot seed lots could have implications when determining the sample sizes and statistical analyses methods required for seed health tests. Although the pathogen distribution on individual seeds in most cases was nonnormal, as has been demonstrated for other bacteria-plant associations in the aforementioned studies, differences in the distribution patterns and incidence were also observed between lots. Of note, among seed lots with similar levels of infestation (CFU per gram bulk assay), the rate of incidence observed was markedly different from lot to lot. For example, the incidence of individual seeds infested with $X$. hortorum pv. carotae ranged from 7

Table 5. Best-fit probability distributions of Xanthomonas hortorum pv. carotae populations recovered from individual carrot seeds and their associated Kolmogorov-Smirnov and Anderson-Darling goodness-of-fit test statistics ${ }^{\mathrm{a}}$

\begin{tabular}{crlcc}
\hline $\begin{array}{l}\text { Year, } \\
\text { seed lot }\end{array}$ & $\boldsymbol{N}^{\mathbf{b}}$ & Fitted distribution & $\begin{array}{c}\text { Kolmogorov- } \\
\text { Smirnov }\end{array}$ & $\begin{array}{c}\text { Anderson- } \\
\text { Darling }\end{array}$ \\
\hline 2014 & & & & \\
1 & 30 & Log-Pearson 3 & 0.1717 & 0.7928 \\
2 & 4 & ND & ND & ND \\
3 & 33 & Weibull & 0.0939 & 2.2108 \\
4 & 28 & Dagum (4P) & 0.1491 & 25.6740 \\
5 & 12 & General $\gamma(4 \mathrm{P})$ & 0.1548 & 14.7560 \\
6 & 4 & ND & ND & ND \\
7 & 7 & Power Function & 0.1383 & 8.8306 \\
8 & 7 & Log- $\gamma$ & 0.3021 & 0.9899 \\
9 & 16 & Pareto 2 & 0.1672 & 0.5119 \\
10 & 6 & Error & 0.2569 & 0.4883 \\
2015 & & & \\
11 & 50 & Weibull (3P) & 0.1251 & 11.8360 \\
12 & 26 & Log- $\gamma$ & 0.1148 & 0.4548 \\
13 & 8 & Levy & 0.1734 & 0.36482 \\
14 & 18 & Log-Pearson 3 & 0.2267 & 17.2610 \\
15 & 6 & $\gamma(3 \mathrm{P})$ & 0.1864 & 6.3271 \\
16 & 0 & ND & ND & ND \\
17 & 0 & ND & ND & ND \\
2016 & & & & \\
18 & 15 & Geometric (discrete) & 0.2732 & 1.9374 \\
19 & 31 & Pearson 6 (4P) & 0.1728 & 34.1810 \\
20 & 10 & General $\gamma(4 \mathrm{P})$ & 0.1533 & 11.9210 \\
21 & 43 & Burr & 0.0612 & 0.1834 \\
22 & 47 & Burr & 0.0846 & 0.3578 \\
23 & 44 & Phased Bi-Exponential & 0.1201 & 4.9719 \\
24 & 30 & Log-Logistic (3P) & 0.1363 & 23.1790 \\
\hline
\end{tabular}

${ }^{\text {a }} \mathrm{ND}=$ not determined due to low sample size.

${ }^{\mathrm{b}}$ Number of Xanthomonas hortorum pv. carotae-positive seeds detected in the seed lot. 
to $94 \%$ among the seed lots that were found to be infested at approximately $1 \times 10^{7} \mathrm{CFU} / \mathrm{g}$ of seed using the bulk (10-g) seed wash assay. Depending on how many seeds are actually infested in a seed lot, it is likely that sample sizes for bulk seed wash assays would need to be adjusted to increase the chances of including relatively few, high-titer seeds or a sufficient number of low-titer seeds in order to achieve the desired level of statistical significance. A nonnormal distribution of $X$. hortorum pv. carotae in carrot seed could also affect the number of seeds needed to perform meaningful seedling grow-out assays, or the amount of time for symptoms to develop from seedborne inoculum (Dutta et al. 2012, 2016; Gitaitis and Nilakhe 1982).

A low frequency of large pathogen populations on seed may also have postharvest consequences on disease-free seed in the same seed lot. For instance, it is not known whether secondary transmission of $X$. hortorum pv. carotae can occur from infested to noninfested seed within a seed lot, as has been demonstrated for X. euvesicatoria in pepper (Dutta et al. 2016). Highly infested seed in seed lots may also be more difficult to disinfest using hot water or other seed treatments, especially when they represent just a few seeds in a large seed lot. As a result, the quality of the entire seed lot can be negatively affected due to repeated treatments targeting a relatively small proportion of seed in the lot.

The incidence of infested seed in a seed lot and the X. hortorum $\mathrm{pv}$. carotae populations found on individual seed are likely important factors influencing seedborne transmission in carrot root crops, as has been observed in previous studies with other bacterial plant pathogens (Gitaitis and Nilakhe 1982; Roberts et al. 1999; Schaad 1982). Although $X$. hortorum pv. carotae was not detected from the majority $(65.6 \%)$ of individual seeds assayed from commercial seed lots produced in central Oregon, and 9 of the 24 seed lots tested contained $20 \%$ or less infested seed overall, a relatively few number of highly infested seeds could provide sufficient inoculum to cause bacterial blight outbreaks in carrot root crop production. For Pseudomonas savastanoi pv. phaseolicola, it was determined that 1 infected seed in 20,000 was sufficient to cause epidemics under favorable disease conditions (Trigalet and Bidaud 1978) and, for some diseases, a zero tolerance has been recommended (Dutta et al. 2012). Seed contamination thresholds for the development of bacterial blight were investigated by Umesh et al. (1998), who concluded that $1 \times 10^{4} \mathrm{CFU} / \mathrm{g}$ of seed was enough for disease development and $1 \times 10^{5} \mathrm{CFU} / \mathrm{g}$ of seed was able to cause yield reductions under irrigated production conditions in California. However, relatively little work has been conducted to investigate the effect of pathogen frequency and population levels associated with individual seeds on seed-to-seedling transmission and epidemic development (Dutta et al. 2012; Roberts et al. 1999). Further research should focus on determining the implications of the presence of a relatively few, highly infested seeds in carrot seed lots on the detection, epidemiology, and management of bacterial blight in carrot production.

\section{Acknowledgments}

We thank J. Chang and K. Johnson for providing valuable reviews to the manuscript; and T. Belvoir, S. Downing, K. Duggan, and T. Klopp for providing their technical assistance.

\section{Literature Cited}

Asma, M. 2005. Proposal for a new method for detecting Xanthomonas hortorum pv. carotae on carrot seeds. ISTA Method Validation Rep. 2:1-17.

Butler, M., and Simmons, R. 2013. 2013 Agricultural Statistics: Central Oregon. https://agsci.oregonstate.edu/coarec

du Toit, L. J., Crowe, F. J., Derie, M. L., Simmons, R. B., and Pelter, G. Q. 2005. Bacterial blight in carrot seed crops in the Pacific Northwest. Plant Dis. 89:896-907. du Toit, L. J., Derie, M. L., Christianson, C. E., Hoagland, L., and Simon, P. 2014 First report of bacterial blight of carrot in Indiana caused by Xanthomonas hortorum pv. carotae. Plant Dis. 98:685.

Dutta, B., Block, C., Stevenson, K., Sanders, F. H., Walcott, R., and Gitaitis, R. 2013. Distribution of phytopathogenic bacteria in infested seeds. Seed Sci. Technol. 41:383-397.

Dutta, B., Gitaitis, R. D., Smith, S., Van Keith, S., and Langston, D. 2016 Pathogen distribution, incubation period and seedling transmission resulting from secondary contamination of pepper seeds with Xanthomonas euvesicatoria. Seed Sci. Technol. 44:104-113.

Dutta, B., Scherm, H., Gitaitis, R., and Walcott, R. 2012. Acidovorax citrulli seed inoculum load affects seedling transmission and spread of bacterial fruit blotch of watermelon under greenhouse conditions. Plant Dis. 96: 705-711.

Gilbertson, R. L. 2002. Bacterial leaf blight of carrot. Pages 11-12 in: Compendium of Umbelliferous Crop Diseases. American Phytopathological Society, St. Paul, MN.

Gitaitis, R. D., and Nilakhe, S. S. 1982. Detection of Xanthomonas campestris pv. vignicola in southern pea seed. Plant Dis. 66:20-22.

Gugino, B. K., Carroll, J. E., Chen, P., Ludwig, J. W., and Abawi, G. S. 2004 Carrot Leaf Blight Diseases and Their Management in New York. Cornell Cooperative Extension IPM Disease Identification Sheet, Geneva, NY, U.S.A.

Hirano, S. S., Nordheim, E. V., Arny, D. C., and Upper, C. D. 1982. Lognormal distribution of epiphytic bacterial populations on leaf surfaces. Appl. Environ. Microbiol. 44:695-700.

Ishimaru, C., Eskridge, K. M., and Vidaver, A. K. 1991. Distribution analyses of naturally occurring epiphytic populations of Xanthomonas campestris pv. phaseoli on dry beans. Phytopathology 81:262-268.

Kendrick, J. B. 1934. Bacterial blight of carrot. J. Agric. Res. 49:493-510.

Loper, J. E., Suslow, T. V., and Schroth, M. N. 1984. Lognormal distribution of bacterial populations in the rhizosphere. Phytopathology 74:1454-1460.

Meng, X. Q., Umesh, K. C., Davis, R. M., and Gilbertson, R. L. 2004 Development of PCR-based assays for detecting Xanthomonas campestris pv. Carotae, the carrot bacterial leaf blight pathogen, from different substrates. Plant Dis. 88:1226-1234.

Miller, S. A. and Lewis Ivey, M. L. 2006. Hot water treatment and chlorine treatment of vegetable seeds to eradicate bacterial plant pathogens. Ohio State University Extension Fact Sheet HYG-3085-05.

Myung, I.-S., Yoon, M.-J., Lee, J.-Y., Kim, G.-D., Lee, M.-H., Hwang, E.-Y., and Shim, H. S. 2014. First report of bacterial leaf blight of carrot caused by Xanthomonas hortorum pv. carotae in Korea. Plant Dis. 98:275.

Nishiyama, K., Fukunishi, T., Terada, T., and Ezuka, A. 1979. Bacterial blight of carrot Daucus carota var. sativa caused by Xanthomonas carotae a bacterial disease new to Japan. Ann. Phytopathol. Soc. Jpn. 45:683-688.

Pruvost, O., Boyer, C., Robène-Soustrade, I., Jouen, E., Saison, A., Hostachy, B., and Benimadhu, S. 2010. First report of Xanthomonas hortorum pv. carotae causing bacterial leaf blight of carrot in Mauritius. Plant Dis. 94: 1069.

Roberts, S. J., Hiltunen, L. H., Hunter, P. J., and Brough, J. 1999. Transmission from seed to seedling and secondary spread of Xanthomonas campestris pv. campestris in Brassica transplants: Effects of dose and watering regime. Eur. J. Plant Pathol. 105:879-889.

Russell, T. S. 1988. Inoculum thresholds of soilborne pathogens: Some aspects of sampling and statistics in seed sampling and establishment of threshold levels Phytopathology 78:880-881.

Schaad, N. W. 1982. Detection of seedborne bacterial plant pathogens. Plant Dis 66:885-890.

Shapiro, S. S., and Wilk, M. B. 1965. An analysis of variance test for normality (complete samples). Biometrika 52:591-611.

Starr, M. P., and Stephens, W. L. 1964. Pigmentation and taxonomy of the genus Xanthomonas. J. Bacteriol. 87:293-302.

Trigalet, A., and Bidaud, P. 1978. Some aspects of epidemiology of bean halo blight. Pages 895-902 in: Proc. IVth Int. Conf. Plant Pathogenic Bacteria, Vol. II. Sta. Path. Veg. Phytobact., INRA.

Umesh, K., Davis, R., and Gilbertson, R. 1998. Seed contamination thresholds for development of carrot bacterial blight caused by Xanthomonas campestris pv. carotae. Plant Dis. 82:1271-1275.

Williford, R. E., and Schaad, N. W. 1984. Agar medium for selective isolation of Xanthomonas campestris pv. carotae from carrot seeds. (Abstr.) Phytopathology $74: 1142$ 